\title{
L'intérêt historiographique des manuscrits de travail de linguistes : l'exemple de la linguistique générale de Joseph Vendryes
}

\author{
Pierre-Yves Testenoire \\ Université Paris-Sorbonne, Histoire des Théories Linguistiques \\ pytestenoire@yahoo.fr
}

Résumé. Dans quelle mesure les manuscrits de travail des linguistes présentent-ils un intérêt pour les historiens de la linguistique ? Ce type de documents suscite, depuis quelques années, un regain indéniable de curiosité. L'exploration d'un nombre croissant de fonds d'archives de savants permet, au-delà d'un élargissement de l'horizon documentaire, d'envisager des approches comparatives entre différentes pratiques savantes. Elle ouvre aussi de nouvelles interrogations. Cet article réfléchit à l'intérêt historiographique de ce type de documents à partir d'un cas inédit : les papiers de travail consacrés à la linguistique générale du linguiste français, Joseph Vendryes (1875-1960). On met en évidence le double intérêt des archives privées de savants : documentaire et processuel. On aborde, dans un premier temps, les manuscrits d'un point de vue documentaire de façon à dégager ce que leur prise en compte modifie de notre connaissance de la linguistique générale de Joseph Vendryes. Le deuxième temps, consacré à la perspective processuelle, fait des manuscrits le lieu de l'observation de la méthode. Il s'agit d'analyser, à partir des manuscrits préparatoires à l'écriture d'un article, comment s'élabore un discours scientifique à caractère général sur le langage et les langues. Ces archives privées sont au carrefour de questionnements de recherche qui relèvent aussi bien d'une histoire conceptuelle que d'une histoire sociale des sciences du langage.

\begin{abstract}
To what extent are linguists' work manuscripts of interest to linguistic historians? For several years, these types of documents have brought about an undeniable rise in interest. Beyond expanding the horizon of documentation, exploring a growing number of archival collections from different scholars enables the consideration of comparative approaches between their scholarly practices. It also opens up new questions. This article questions the historiographic interest of this type of document from a new case: the paper works dedicated to general linguistics from the French linguist, Joseph Vendryes (1875-1960). This article aims to highlight the double interest of scholars' private archives: documentation and procedural. In a first phase, it tackles manuscripts from a documentary point of view in order to draw what their inclusion changes in our knowledge of Joseph Vendryes' general linguistics. In a second phase, dedicated to procedural perspective, the manuscripts become the point of observation for the method. It is a question of analysing, from preparatory manuscripts to writing an article, how scientific discourse is created overall in language and speech. These private archives are at the crossroads of research questions that just as well bring up a conceptual history as a social history of language sciences
\end{abstract}


Dans quelle mesure les manuscrits de travail des savants présentent-ils un intérêt pour les historiens de la linguistique ? Si l'exploitation de ce type de documents ne date pas d'hier, elle connaît, ces dernières années, un regain de vigueur indéniable. Aux fonds d'archives connus depuis plusieurs décennies, comme ceux de Saussure (Godel, 1957), de Guillaume (Valin, 1971) ou de Meillet (Bouquet, 1988 et Swiggers, 1991), vient s'ajouter l'exploration récente de nouveaux corpus : les manuscrits de Bally (Forel, 2008), de Benveniste (Fenoglio, 2009, Brunet, 2011), d'Haudricourt (2010), de Tesnière (Chepiga, 2012)... L'ouverture de ces nouveaux fonds d'archives a donné lieu aux premières tentatives d'études contrastives entre les papiers de différents savants (Fenoglio, 2012, Chepiga \& Sofia, 2014) ${ }^{1}$. Cependant, plus que l'élargissement de l'horizon documentaire, les travaux actuels traduisent une innovation dans l'approche méthodologique de ce type de documents. De cette évolution, le traitement du cas emblématique des manuscrits de Saussure est exemplaire.

Le travail scientifique sur les manuscrits du linguiste genevois a été inauguré par les travaux fondateurs de Robert Godel (1957) et de Rudolf Engler (Saussure, 1968-1974). Ils sont rendus possibles dans le courant des années 50 par le dépôt à la Bibliothèque de Genève des manuscrits du linguiste. L'ambition de Godel et d'Engler est de confronter le Cours de linguistique générale (désormais CLG) à ses différentes sources : cahiers d'étudiants et notes autographes. Dans sa visée comme dans sa méthode, la démarche emprunte alors directement à la philologie. Pour restituer un urtext définitivement perdu - le cours oral tel que l'a prononcé Saussure -, les savants collationnent les variantes, élisent un meilleur témoin (les cahiers de Riedlinger pour le deuxième cours par exemple) ${ }^{2}$, pratiquent la lectio difficilior... Établie selon les meilleurs principes et légitimée par l'acribie critique des travaux de Godel et d'Engler, cette «philologie saussurienne » a donné corps, depuis soixante ans, à une spécialisation au sein des études sur Saussure, spécialisation vivace comme le prouvent les nombreuses éditions de textes saussuriens de ces dernières années ${ }^{3}$. Parallèlement à cette tradition philologique, se développe un ensemble de travaux dont les principes et la méthode se réclament de la génétique textuelle.

Initialement fondée pour l'analyse des manuscrits littéraires, la critique génétique s'est élargie au tournant des années 2000 aux manuscrits des sciences dites « exactes » (Barberousse \& Pinon, 2003) et des sciences humaines (D’Iorio \& Ponton, 2003, Fenoglio, 2012). L'approche des manuscrits, dans cette perspective, n'est plus orientée vers l'établissement d'un texte stabilisé, mais s'attache à l'observation des processus d'écriture. La constitution, quand elle est possible, de dossiers de genèse et l'observation des traces laissées par le scripteur sur les manuscrits - ratures, corrections, ajouts... - servent à comprendre la méthode et les problèmes à l'œuvre dans l'écriture d'un article, d'une conférence ou pour l'élaboration d'un concept. Cette perspective de recherches s'illustre dans les premières publications d'études saussuriennes dans des revues de critique génétique (Testenoire, 2010, Sofia, 2012, D’Ottavi, 2012) qui suivent de près les premières applications de la démarche génétique aux manuscrits d'Émile Benveniste (Fenoglio, 2009 et 2012a). Ces deux approches des manuscrits, philologique et génétique, loin de s'opposer, sont complémentaires ; elles correspondent au double intérêt - documentaire et processuel que présente pour l'historien ce type d'écrits.

En tant que documents, les manuscrits de travail des linguistes contiennent des éléments susceptibles d'enrichir la connaissance historique de leurs activités scientifiques. Ils sont précieux pour préciser l'inscription d'un savant dans un champ social et culturel, pour cartographier ses connaissances 
scientifiques ou pour déterminer ses sources et ses influences. La correspondance scientifique ou l'exhumation de projets non publiés jouent, par exemple, ces rôles. Les informations qu'elles fournissent ont un statut différent de celles de l'œuvre publiée et validée par le savant, mais elles les complètent et vont parfois jusqu’à réorienter leurs interprétations.

En tant que témoin d'un processus de travail et d'écriture, les manuscrits constituent aussi des observatoires privilégiés de la science linguistique en train de se faire. Conservant des hypothèses abandonnées, d'autres reprises et transformées, ils s'apparentent à de véritables laboratoires méthodologiques et conceptuels. Surtout, ils gardent la trace des opérations d'écriture à l'œuvre dans l'élaboration d'un discours sur la langue. Ainsi considéré, le manuscrit permet d'appréhender le travail scientifique comme une activité scripturale et discursive. Par là même, ce sont aux spécificités et aux contraintes des différentes pratiques attestées dans l'étude du langage - une enquête de terrain, un travail lexicographique, l'écriture d'une grammaire... - que les manuscrits donnent accès. Y a-t-il, entre les différents fonds d'archives, des récurrences processuelles et méthodologiques dans l'écriture d'un article théorique ou d'un dictionnaire ? Comment celles-ci varient-elles selon les époques ? les aires culturelles ? les situations institutionnelles ? Ce sont ce type de questions que la perspective génétique appliquée aux manuscrits scientifiques pose à l'historien. Le présent article entend illustrer cette double dimension du travail sur les manuscrits de linguistes à partir de l'étude d'un cas inédit : les archives de Joseph Vendryes (1875-1960).

Les manuscrits du linguiste ont été légués au Collège de France à sa mort, en 1960. Conservés au sein de cet établissement, puis temporairement à l'Institut Mémoires de l'édition contemporaine (IMEC), ces manuscrits sont restés inaccessibles pendant une cinquantaine d'années faute de classement. Le classement a été effectué par nos soins en 2011 et 2012 en partenariat avec des archivistes de l'IMEC. Les manuscrits sont désormais librement consultables à la Bibliothèque générale du Collège de France et l'inventaire du fonds à été publié (Testenoire, 2015) ${ }^{4}$. Le fonds d'archives Joseph Vendryes est à contenu exclusivement scientifique; il contient des notes de travail, des documents préparatoires à des articles, des cours ou des conférences ainsi que des lettres reçues de différents collègues. D'autres manuscrits autographes, auxquels nous avons eu accès ${ }^{5}$, sont en possession des descendants du linguiste et se composent, de la même manière, de correspondance et de notes de travail. L'œuvre scientifique de Joseph Vendryes compte une dizaine d'ouvrages et plus de deux cent cinquante articles; elle couvre trois grands domaines : la linguistique générale, la grammaire comparée et les études celtiques ${ }^{6}$. Si la majorité des manuscrits du savant qui sont conservés concernent ce dernier domaine, nous nous concentrerons ici sur ceux relatifs à la linguistique générale.

Notre démarche s'articulera en deux temps, illustrant en cela le double intérêt historiographique de ces manuscrits de travail. Nous aborderons, dans un premier temps, les manuscrits d'un point de vue documentaire de façon à dégager ce que leur prise en compte modifie de notre connaissance de la linguistique générale de Joseph Vendryes. Le deuxième temps sera consacré à la perspective processuelle, en faisant des manuscrits le lieu de l'observation de la méthode. Pour analyser comment s'élabore un discours scientifique à caractère général sur les langues nous nous pencherons sur un cas précis : les manuscrits préparatoires d'un article paru en 1952.

\section{La linguistique générale de Joseph Vendryes : I'apport des manuscrits}

\section{1 État des lieux historiographiques}

Si l'historiographie reconnaît l'apport capital de Joseph Vendryes dans le développement des études celtiques en France, l'importance de sa linguistique générale dans le siècle paraît plus indéterminée. Christian Puech, par exemple, s'interroge :

L'influence de Vendryes sur les générations suivantes est difficile à établir : Vendryes a des successeurs. A-t-il des disciples ? [...] En ce qui concerne la linguistique 
générale, la génération suivante sera en effet beaucoup plus influencée par les écoles périphériques (Cercle Linguistique de Prague, Copenhague...). (2000 : 460).

Plusieurs facteurs contribuent à rendre l'importance de son activité de généraliste délicate à déterminer. L'un d'entre eux tient à ce qu'elle n'a pas produit d'innovation conceptuelle majeure. Piet Desmet et Pierre Swiggers (1996) n'attribuent à Vendryes qu'une innovation terminologique : le couple sémantème / morphème. Les sémantèmes entendus comme «les éléments linguistiques exprimant les idées de représentation » s'opposent aux morphèmes définis comme «ceux qui expriment les rapports entre les idées », dont les catégories grammaticales : genre, nombre, personne, temps, mode... (Vendryes, [1921] 1968 : 92). Cette distinction sera reprise par Charles Bally (1932 : 287-290) et correspond, dans la théorie fonctionnaliste de Martinet, à la distinction entre les deux types de monèmes - lexèmes et morphèmes.

Une seconde difficulté historiographique concerne le problème de l'originalité de sa pensée. Représentant emblématique de la linguistique française de la première moitié du XXe siècle dans son orientation sociohistorique, Vendryes est immanquablement présenté, par ses contemporains comme par les historiens, comme «the follower of Meillet», «son élève», «son collaborateur », voire «son lieutenant » Départager la pensée linguistique de Vendryes de celle de son maître relève de la gageure, tant l'influence de ce dernier est constamment revendiquée. Dans la préface du Langage, Meillet est présenté comme «l'instigateur de l'ouvrage » et son relecteur. Vendryes ajoute : «Puisse le lecteur reconnaître les traces de son influence!»([1921] 1968: 14). La préface du Traité de grammaire comparée des langues classiques est encore plus explicite quant aux convergences théoriques entres les deux linguistes :

\begin{abstract}
Dans un livre écrit en collaboration, le lecteur souhaite généralement de connaître la part qui revient à chacun des collaborateurs. La question est ici un peu oiseuse, en raison de la communion de pensée où ont vécu les deux auteurs depuis près de trente ans qu'ils se sont rencontrés pour la première fois, l'un comme maître, l'autre comme élève. Il n'est guère de question traitée dans ce livre qu'ils n'aient eu l'occasion de discuter souvent entre eux. (Meillet \& Vendryes, 1924 : avant-propos, VI) ${ }^{8}$
\end{abstract}

Du fait de cette «communion de pensée » revendiquée, Le Langage est parfois présenté comme le manuel de linguistique générale que Meillet n'a jamais publié et qu'il aurait sous-traité à Vendryes. La découverte récente dans les archives de Meillet d'un livre de linguistique générale entièrement rédigé mais non donné à la publication (Granucci, 1992 et Meillet, 1995) met à mal cette interprétation. Esquissé en 1909-1910, le livre est rédigé au début des années 20. Il intègre certains apports du CLG, comme la distinction entre synchronie et diachronie qui fournit le plan de l'ouvrage en deux parties, intitulées « linguistique descriptive » et «linguistique historique », mais il présente aussi des similitudes avec le livre de Vendryes dont Meillet a alors connaissance. À titre d'exemple, le plan tripartite adopté par Meillet dans la première partie - «1. Phonétique », «2. Analyse du lexique au point de vue du sens », «3. Principes de morphologie » entendu comme « l'étude des formes grammaticales »-correspond, dans un ordre différent, aux trois premiers chapitres du Langage : «Les sons », «La grammaire » et «Le vocabulaire ». Les différences d'analyse dans le détail révèlent que les deux projets sont indépendants, même s'ils sont mutuellement informés de l'existence, voire du contenu, de l'autre. Ainsi le manuscrit de Meillet compte un long développement consacré à l'analyse de la phrase (Meillet, 1995 : 119-130), qui ne fait pas l'objet d'une étude spécifique chez Vendryes. Cette différence fait écho au principal reproche que Meillet adresse au Langage : "Soit le chapitre sur les catégories grammaticales. Il aurait été possible de distinguer nettement entre l'articulation de la phrase, d'une part, et les notions générales qui s'expriment en même temps que les notions » (Meillet, 1922:8).

Par-delà ces divergences, Vendryes partage avec Meillet une même conception de la linguistique générale. Plutôt qu'une « généralité de principes » (Normand, 2000) telle que la pratique un Victor Henry ou un Saussure, Vendryes souscrit à une conception sommative de la linguistique générale. Elle procède à la fois par induction de généralités à partir d'observations linguistiques et par une démarche encyclopédique, comme celle des Langues $d u$ Monde dont Vendryes rédige la partie consacrée aux langues indo-européennes. Cette conception sommative voue le «traité de linguistique générale » à devenir un horizon programmatique inaccessible et condamne ses tentatives de rédaction soit à la nonpublication (Meillet), soit à n'en être qu'une introduction (Vendryes). 
Une autre difficulté d'appréhension de la pensée théorique de Vendryes réside dans le sort ambivalent qu'a connu Le Langage, ce que l'on pourrait appeler sa fortune et son infortune éditoriales. Son infortune éditoriale est due à la Grande Guerre qui diffère sa publication. Écrit entre 1911 et 1914, l'ouvrage n'est donné à l'impression qu'au début des années 20. Entretemps paraît le Cours de linguistique générale. Ce télescopage paraît suffisamment important pour que Vendryes en fasse état dans sa préface. S'excusant de présenter «un ouvrage vieux de sept ans déjà », il signale la parution du $C L G$, même s'il ajoute qu'il ne constitue pas, à ses yeux, «un exposé méthodique et complet de linguistique générale » (Vendryes, [1921] 1968 : 13-14). La réception immédiate associe Le Langage aux livres de Sapir (1921) et de Jespersen (1922), aux titres identiques, qui paraissent en même temps ${ }^{9}$; Antoine Meillet, par exemple, leur consacre un compte rendu commun. À titre rétrospectif, le langage fait partie de cet ensemble plus large de traités de linguistique générale qui paraissent à un rythme croissant entre le dernier quart du XIXe siècle et les années 20 (v. Auroux, 1988 et 2000).

La fortune éditoriale du Langage tient, quant à elle, à la variété des langues mobilisées et à la clarté de son propos. Rédigé sans termes techniques, le livre est reçu comme le «traité de linguistique générale attendu par les étudiants et le public » (Meillet, 1922: 8). Ses qualités pédagogiques, unanimement saluées, font son succès public, dont témoignent les multiples rééditions et les nombreuses traductions : anglaise (1925), espagnole (1925), russe (1937), japonaise (1938), arabe (1950), polonaise (1956) et turque $(2002)^{10}$. En France, l'enseignement de linguistique générale à l'École Normale Supérieure dont Vendryes a la charge de 1920 à 1936 contribue à imposer Le Langage comme le manuel de référence pour l'introduction à la linguistique. Dépassant l'audience des seuls spécialistes, l'ouvrage touche les sphères intellectuelles de l'entre-deux guerres, dont des écrivains comme Blaise Cendrars ou Raymond Queneau $^{11}$. Cependant, la réédition jusqu'en 1968 d'un ouvrage achevé en 1914 a pu donner l'illusion du figement d'une pensée datée et faire écran à ses évolutions durant toutes ces années. C'est précisément cette trajectoire du généraliste que le fonds des manuscrits contribue à éclairer.

La production de linguistique générale de Vendryes ne se réduit pas au seul Langage. Le souci de la constitution d'une linguistique générale traverse l'œuvre de Joseph Vendryes depuis son premier article d'envergure, de 1902, sur les lois phonétiques jusqu'aux articles, moins connus des années 50, que nous aurons l'occasion d'aborder. Cet itinéraire est marqué par une constance - la fidélité au programme de recherche formulé par Antoine Meillet - et par des évolutions. L'une des plus notables est celle qui intervient au début des années 30 sans doute au contact des travaux du Cercle de Prague. Alors que sa réflexion était jusque là dominée par les questions d'évolution et de progrès linguistiques - le sous-titre du Langage n'est autre qu'Introduction linguistique à l'histoire -, son activité de généraliste s'oriente vers une approche exclusivement synchronique, à tel point que Greimas dans L'actualité du saussurisme, en 1956, le qualifie de «"synchroniste" intransigeant »(Greimas, 1956 : 203).

\subsection{La linguistique générale de Joseph Vendryes d'après les manuscrits}

Les manuscrits de travail de Joseph Vendryes qui nous sont parvenus complètent, sur bien des points, notre connaissance de son activité de généraliste. C'est le cas, en particulier, d'un ensemble de documents, écrits en janvier 1941, où le linguiste revient lui-même sur son itinéraire et la place qu'y occupe la linguistique générale. Les évènements sombres auxquels Vendryes est alors directement confronté en tant que doyen de la faculté des lettres de Paris - l'évacuation de la Sorbonne en juin 1940, sa fermeture puis sa réouverture par l'occupant à l'automne, l'expulsion des professeurs juifs de l'Université en décembre ${ }^{12}-$ le conduisent à rédiger deux textes autobiographiques. L'un est un testament adressé à sa famille ; l'autre est une longue note écrite à l'attention du secrétaire perpétuel de l'Académie des Inscriptions et Belles-Lettres d'alors, René Dussaud, en prévision de sa nécrologie. Vendryes y décrit son projet en ces termes :

Je voudrais mettre par écrit quelques indications biographiques et tracer de moi en toute objectivité un portrait moral, qui fera connaître, sinon ce que j'ai été, du moins ce que j'aurais souhaité et ce que je me suis efforcé d'être. (Vendryes, janvier 1941, manuscrit communiqué par Georges Vendryes) ${ }^{13}$ 
Dans cet exercice de réflexivité courant sur plusieurs pages, on retiendra cet autoportrait intellectuel :

J'ai un goût naturel pour la flânerie ; j'aime à rêver, mais un peu au hasard et sans plan arrêté. Quand je lis, je m'attache surtout au détail qui m'attire et me retient. Je n'ai pas d'aptitude aux abstractions et aux synthèses, c'est à dire que je n'ai pas l'esprit mathématique, ni philosophique. Je vois bien le concret: grâce à une mémoire prompte et fidèle, $j$ 'en retiens aisément l'image dans mon souvenir, et j'en tire volontiers des motifs d'association et de comparaison. Or, par l'examen du concret, on est tenté de s'étendre toujours en surface, au détriment de la profondeur. Je n'ai pas de facilité naturelle à produire. J'aime que les choses soient bien mises en ordre, disposées clairement et exactement exprimées. Mais la rédaction m'est difficile : je réussis rarement du premier jet; je dois m'y reprendre à plusieurs fois et raturer beaucoup pour obtenir un résultat qui me satisfasse.(Ibid.)

S'il convient de faire la part de l'humilité, feinte ou sincère, inhérente à ce type d'écrit, l'autoportrait a aussi valeur de bilan épistémologique. Il dessine un tempérament scientifique plus porté vers la dispositio que vers l'inventio. Les qualités que Vendryes se reconnaît - celle d'être un bon pédagogue à défaut d'être un grand théoricien - concordent avec les caractéristiques qu'on attribue à ses écrits de linguistique générale: leur clarté et leur absence de formalisme. Dans cet attachement au «concret», c'est l'empirisme de ses travaux qui se trouve réaffirmé. Le propos se poursuit d'ailleurs par un bilan critique de sa production de linguistique générale. Le Langage est assez vite évoqué :

Mon livre sur le Langage, dont le prodigieux succès reste pour moi un sujet d'étonnement, n'est au fond qu'une collection de faits de détail que j'ai choisis et classés de mon mieux. S'il a fait connaître au grand public ce qu'est notre science et suscité quelques vocations de linguistes, il n'aura pas été inutile. (Ibid.)

Vendryes aborde plus longuement les projets non publiés :

J'aurais voulu laisser un ouvrage d'ensemble sur la linguistique, où j'aurais exposé tous les aspects de cette science si variée. J'ai sur ce sujet des notes abondantes, et j'y ai consacré des cours successifs. (Ibid.)

Le regret touche à l'écriture d'un «traité de linguistique générale », projet que la préface du Langage présentait pourtant comme «une entreprise périlleuse », voire comme un idéal inaccessible (Vendryes [1921] 1968 : 13). Les cours dont il est question renvoient vraisemblablement à l'enseignement de linguistique générale que Vendryes dispense à l'Ecole Normale Supérieure de 1920 à 1936. Quant « aux notes abondantes » sur le sujet, elles n'ont pas été retrouvées. Si elles indiquent pour cet « ouvrage d'ensemble » de la documentation, rien ne certifie une rédaction ou une ébauche de rédaction. Tel n'est pas le cas en revanche du second projet évoqué :

J'ai également amassé la matière d'un livre sur l'esthétique du langage. J'en avais même rédigé quelques morceaux. Les ayant soumis à l'examen d'un ou deux intimes, suivant une précaution que j'ai prise avec tous mes ouvrages, même les plus menus, je me suis rendu compte que leur approbation manquait de chaleur et de spontanéité et qu'elle masquait des réserves. Aussi me suis-je abstenu de continuer à mettre par écrit des idées insuffisamment mûries. Je n'ai pas cessé d'y réfléchir et même d'augmenter les notes qui s'y rapportent, au fur et à mesure de mes lectures. Mais je doute que ma besogne administrative, qui accapare de plus en plus mon activité, me laisse le loisir nécessaire à fixer une conception d'ensemble et une rédaction définitive. (Ibid.)

La rédaction inachevée du livre sur l'esthétique du langage peut, en partie, être datée grâce à la correspondance. On trouve, en effet, dans les lettres de Joseph Vendryes à Antoine Meillet quatre mentions de ce projet. La première se situe le 2 septembre 1928 :

Nos vacances se poursuivent dans le calme le plus parfait. Je mène une vie des plus régulières, sortant très peu de la maison, et passant la plus grande partie de mon temps à faire du gallois : je consacre le reste à l'Esthétique du langage, mais je ne suis pas satisfait du tour que le cela prend. (Vendryes, lettre à Meillet datée du 2 septembre 1928, Collège de France, MLT 14.31). 
Le sujet revient quatre jours plus tard :

Je profite de ces belles journées de vacances calmes pour travailler. J'espère pouvoir vous soumettre à la rentrée un bon morceau de mon Esthétique du Langage. Cela formera un petit volume pour la Collection philosophique d'Alcan ${ }^{14}$. J'en ai parlé à Delacroix ${ }^{15}$ qui m'a promis de le faire accepter sans difficulté. Le sujet est immense et touche à toute la linguistique. Le difficile est de faire tenir l'essentiel en $150\langle$ ou 200〉 pages d'impressions. Je réduis le plus que je peux. (Vendryes, lettre à Meillet datée du 6 septembre 1928, communiquée par Georges Vendryes)

Une lettre du 3 octobre, un mois plus tard, semble y faire de nouveau allusion : «Quand nous nous verrons, j'aurai d'autres morceaux de ma prose à vous soumettre ». Enfin, le projet est de nouveau évoqué, à un état plus avancé, l'été suivant. Dans une lettre du 8 août 1929, Vendryes écrit :

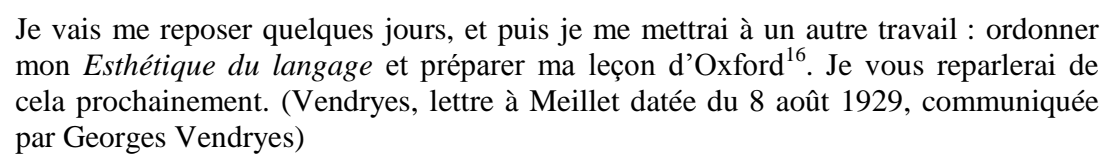

Ces quatre passages confirment - car on s'en doutait - qu'Antoine Meillet est l'un de ces « intimes » évoqués en 1941 dont les « réserves » ont entraîné l'abandon de la rédaction. Malheureusement, alors que Vendryes parle de «nombreuses notes » et de «morceaux rédigés », aucun manuscrit conservé dans le fonds du Collège de France ou en possession de la famille ne paraît directement se rattacher à cet Esthétique du langage.

On trouve, en revanche, des échos de ce projet dans l'œuvre publiée : dans le texte d'une conférence intitulée «La phonologie et la langue poétique » que Vendryes donne, en juillet 1935 , au $2^{\mathrm{e}}$ congrès international de phonétique de Londres. La conférence s'ouvre en ces termes : «Un des mérites de la phonologie, et non le moindre, sera d'avoir ramené dans les cadres de la linguistique l'étude de l'esthétique du langage » ([1935] $1951: 51)^{17}$. Vendryes inscrit son travail sur l'esthétique du langage dans la prolongation de trois chercheurs : Maurice Grammont, Roman Jakobson et Jan Mukarovsky. L'expression «fonction esthétique », qu'il utilise dans sa conférence de 1935 emprunte surtout aux travaux de ce dernier, alors qu'il ne les connaissait pas en 1928-1929 lorsqu'il travaille sur son Esthétique du langage :

La tâche de la phonologie est donc d'étudier les fonctions linguistiques des oppositions phoniques, et parmi ces fonctions, il faut naturellement compter la fonction esthétique, qui a sa place dans toutes les langues et une place prépondérante dans les langues civilisées. (Ibid. : 52)

Vendryes propose, dans la suite de la conférence publiée, d'«assigner deux tâches à la phonologie appliquée à la fonction esthétique », deux tâches qu'il calque sur la distinction saussurienne langue / parole : «l'une consisterait à étudier dans chaque langue les oppositions phoniques capables de produire un effet esthétique; l'autre, à étudier l'utilisation qu'ont faite de ces oppositions les divers poètes » (Ibid.). La suite du texte est consacrée à souligner les décalages entre la phonologie de la langue poétique et celle de la langue parlée contemporaine (par ex. l'expression du digamma dans les poèmes homériques ou du e muet dans des vers français classiques). On peine néanmoins à saisir ce que serait, d'après ce texte, cette phonologie de la parole poétique, notamment à cause du flou qui entoure l'acception de phonologie. Troubetzkoy, dans ses lettres à Jakobson, écrit à propos de cette conférence à laquelle il a assisté, que «son explication (de la phonologie) était aussi indigente qu'inintelligible » (Troubetzkoy, 2006 : 394). Vendryes, il est vrai, y définit le phonème comme « le son produit par l'appareil phonateur de l'homme » (Vendryes [1935] $1951: 51$ ) loin de la définition proposée par les Praguois dès $1930^{18}$. Le projet sur l'esthétique du langage de Vendryes semble avoir été tiraillé par le débat autour de la phonologie qui agite le début des années 30. Principalement redevable, dans un premier temps, aux travaux de Maurice Grammont sur le vers français, la recherche de Vendryes paraît influencée, à partir du début des années 30, par les premiers articles de Mukarovsky parus en français dans les Travaux du Cercle Linguistique de Prague - «Rapports de la ligne phonique avec l'ordre des mots dans les vers 
tchèques » (1929) et surtout «La phonologie et la poétique » (1931). Vendryes prévoit d'ailleurs, dans un premier temps, de publier le texte de sa conférence dans les Travaux du Cercle Linguistique de Prague ${ }^{19}$.

On ne peut pas déduire de la conférence de 1935 la teneur exacte des manuscrits de l'Esthétique du Langage auxquels Vendryes pense encore en 1941. Ce travail, en tout état de cause, ne reflète pas uniquement son goût pour les belles lettres. Vendryes s'intéresse aux productions poétiques, non pas en amateur éclairé, mais bien en linguiste. Cet aspect de ses recherches fait écho aux travaux de poétique d'autres linguistes que l'étude des manuscrits a précisément permis de révéler : les cahiers d'anagrammes dans le cas de Saussure (Starobinski, 1971, Testenoire, 2013), le projet d'article sur la langue de Baudelaire dans le cas de Benveniste (Benveniste, 2011). Si ces trois linguistes développent, chacun avec opiniâtreté, des approches de la langue poétique qui s'adossent à un projet de linguistique générale, il est surtout remarquable qu'aucune d'entre elles n'aboutisse à une publication. Au-delà des circonstances qui peuvent dans chaque cas l'expliquer, une raison plus structurelle semble jouer dans ces abandons : une difficulté, liée à l'organisation des savoirs dans l'Université française, à articuler dans le champ de la réflexion linguistique la problématique du poétique. À titre plus anecdotique, Antoine Meillet se retrouve avec l'Esthétique du langage dans une position qui lui est familière : contrôleur externe des travaux de poétique de Vendryes, vingt ans après avoir contrôlé ceux de Saussure.

\section{Comment généraliser? Le cas de l'article Sur la dénomination}

Venons-en maintenant à l'étude de manuscrits préparatoires de travaux de linguistique générale contenus dans le fonds Vendryes. Pour étudier la méthode du linguiste, nous nous intéresserons au dossier préparatoire de l'article Sur la dénomination paru en 1952 dans le Bulletin de la Société de linguistique de Paris. L'article en question porte sur la dénomination définie comme « le rapport qui existe entre le nom et son objet » (Vendryes, 1952:3). L'objet de cet article est de défendre l'arbitraire du signe plus de dix ans après les objections de Pichon et de Benveniste. La thèse développée par Vendryes est que la contestation de l'arbitraire saussurien repose sur un malentendu et témoigne d'une incompréhension du « point de vue sociologique » qui sous-tend, chez Saussure, le qualificatif « arbitraire ».

Le dossier préparatoire de cet article est assez mince : il comprend 14 feuillets écrits auxquels s'ajoutent 2 documents imprimés. Le tout a été réuni par le linguiste dans une enveloppe qui porte un tampon postal de juillet 1952 sur laquelle le linguiste a écrit : «Fiches utilisées. Pour la Dénomination » (v. fig. 1). 


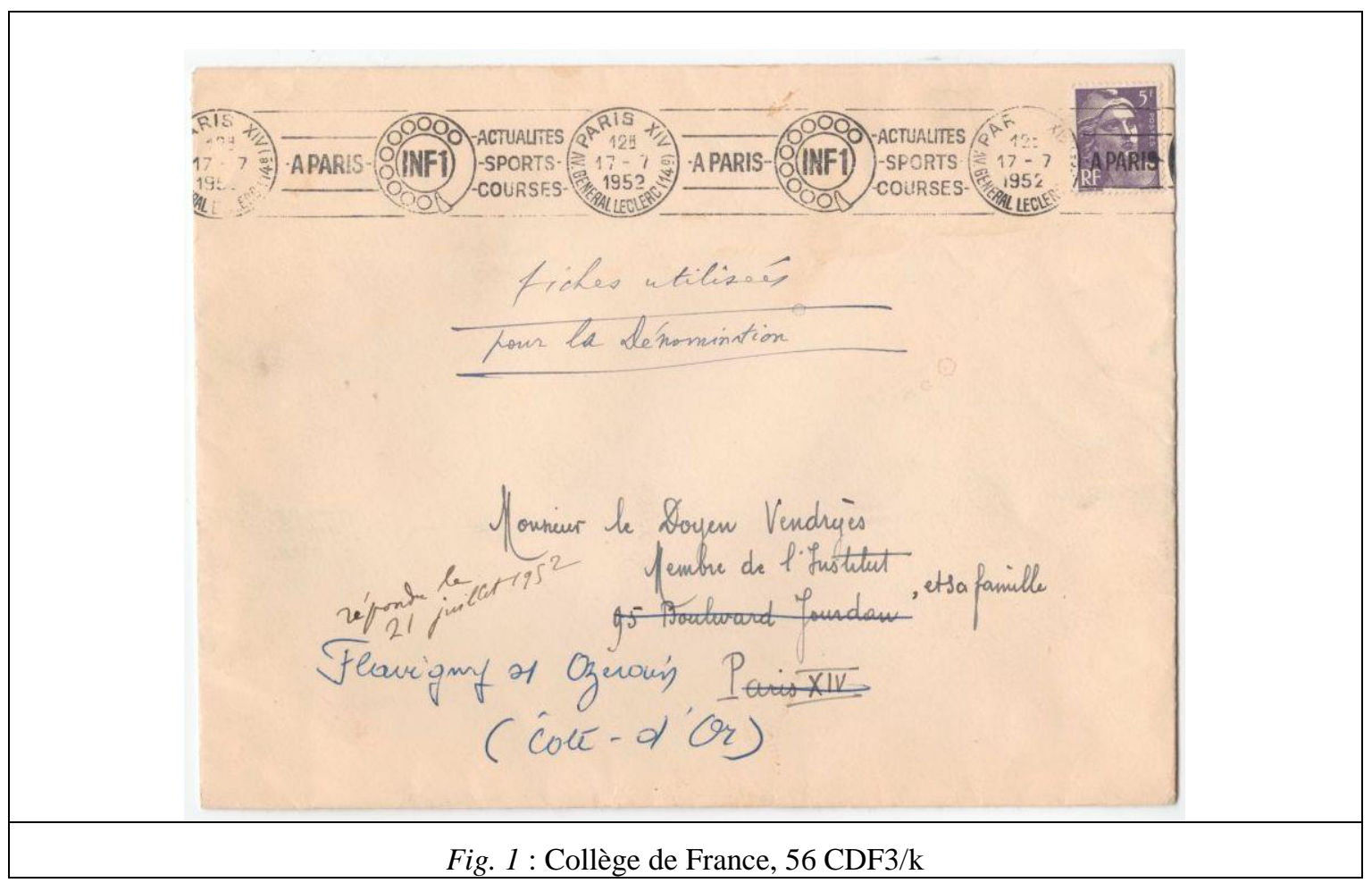

Réunir dans des enveloppes de différents formats les manuscrits préparatoires d'un article est une pratique dûment attestée dans les archives de Vendryes. Les notes sont le plus souvent désignées sur l'enveloppe selon le patron employé ici : le titre de la publication suivi ou précédé des mentions « Fiches utilisées », « Notes à garder », ou encore «À conserver ». Sur certaines enveloppes les finalités de la conservation des documents de travail sont explicitement indiquées. On trouve ainsi : « Notes diverses à garder pour Langue et Parole et pour d'autre articles. ", « ̀̀ garder pour additions à des publications précédentes. », « À garder pour publications en cours »... L'archivage par le linguiste de ses manuscrits en prévision de réutilisations potentielles explique les lacunes des dossiers avec lesquelles il faut composer. Dans le cas présent, les phases de rédactions sont jetées ; ne sont conservés que les rebuts, ce qui n'apparaît mobilisé qu'à titre indirect dans le texte définitif et qui pourra par conséquent resservir : documentation, notes de lecture, faux départs, etc.

Le terme de «fiche » qu'utilise ici Vendryes pour désigner ses matériaux de travail mérite qu'on s'y arrête. La «fiche » dénote, d'après le Trésor de la Langue Française un «petit carton ou [une] feuille rigide, généralement de dimensions normalisées, sur lequel sont inscrits des renseignements déterminés qu'on classe par ordre alphabétique ou méthodique ». C'est un instrument de recherche flexible, permettant l'organisation et les réaménagements de données sur un temps long, dont l'usage savant se répand tout au long du XIXe siècle (Waquet, 2015 : 74-89). Le fonds Vendryes contient, par exemple, quantité de fiches pour des projets lexicographiques sur les langues celtiques. Cependant les manuscrits préparatoires de Sur la dénomination contenus dans l'enveloppe ne sont pas à proprement parler des fiches au format standardisé. Cette appellation utilisée pour désigner ses propres papiers de travail fait écho à cet art de disposer et de classer les faits qui caractérise, selon Vendryes, son travail.

Si l'on ouvre à présent l'enveloppe, on distingue dans le dossier six ensembles textuels, six rédactions continues de taille variable : la plus courte occupe une page, la plus longue en occupe quatre et court sur trois feuillets. En tête du dossier, figurent deux documents imprimés : deux articles de journaux que Vendryes a découpés et conservés. Les deux articles, parus dans Le Monde en janvier 51, abordent des sujets linguistiques. Le premier (fig. 2) porte sur le dictionnaire des termes latins correspondant aux vocables et expressions modernes d'un hiérarque du Vatican, Antonio Bacci, et qui permet de traduire en 
latin «bombe atomique », « radar», «bazooka ». Le second (fig. 3) porte sur la publication au journal officiel d'une loi sur l'enseignement des langues et dialectes locaux.

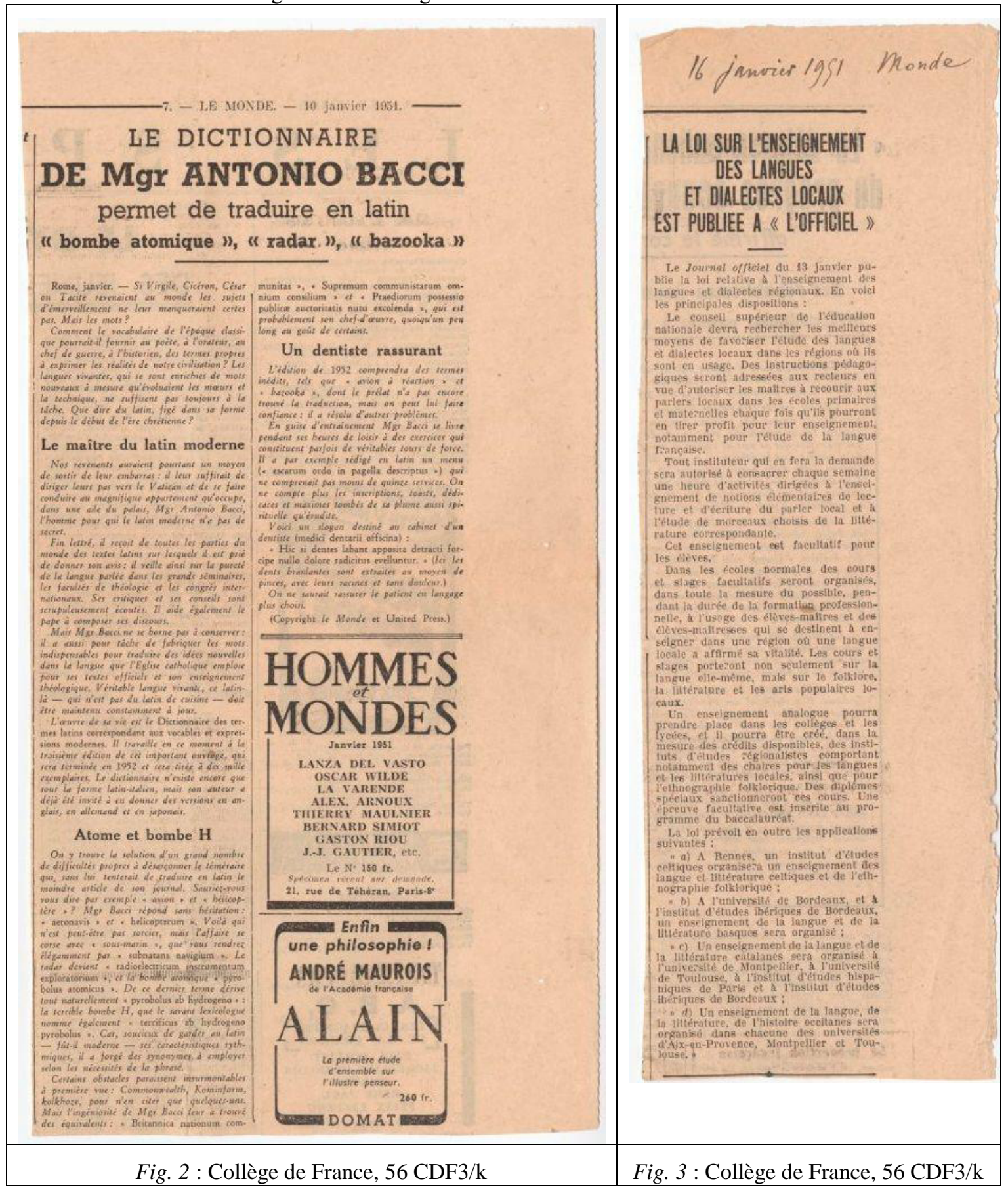

Entre ces deux coupures de presse, figure un feuillet de petit format (fig. 4) contenant un aphorisme de la main de Vendryes : « ce qui est sans mémoire ne saurait être éducatif » : 


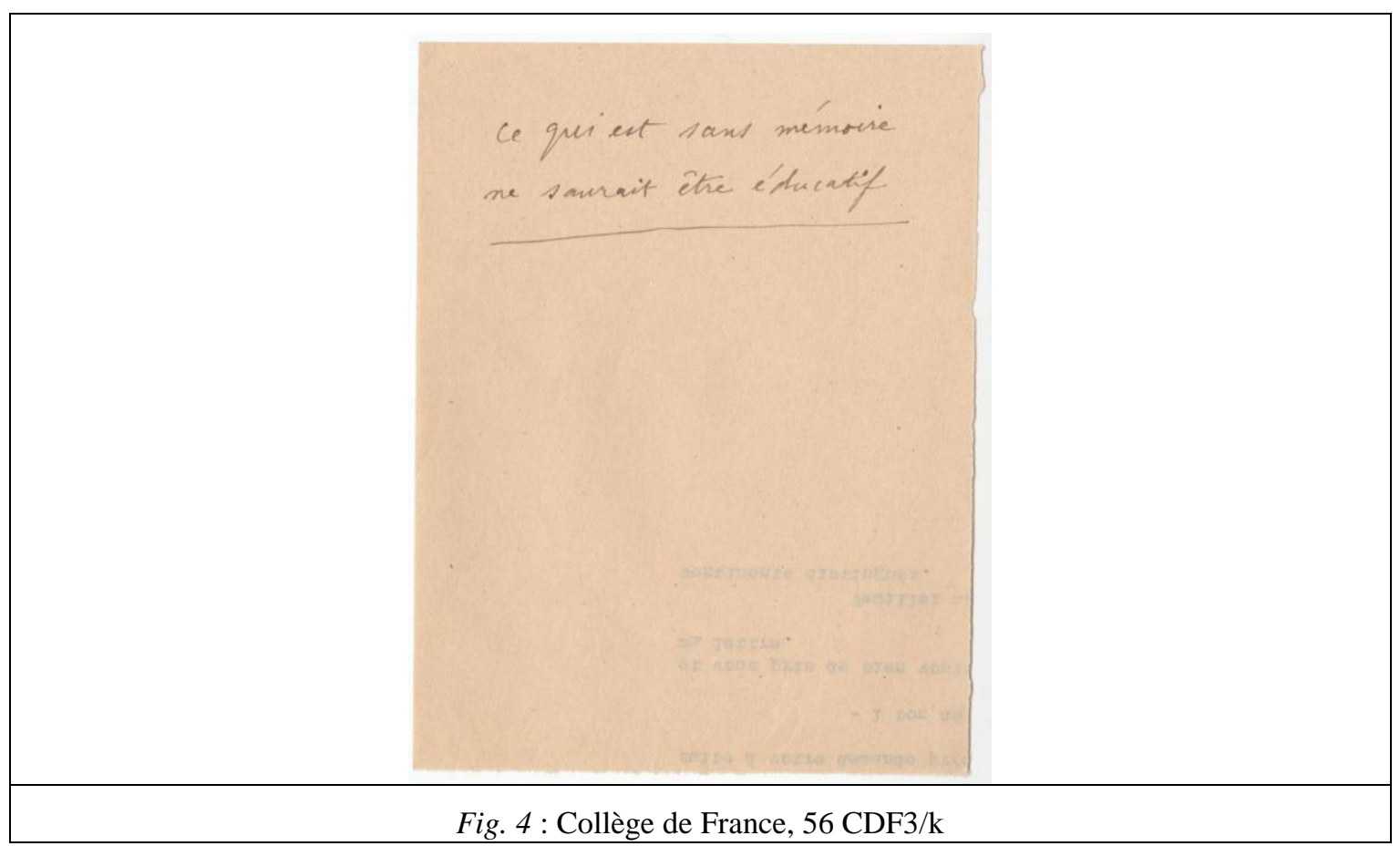

Si de tels aphorismes sont fréquents dans les manuscrits de Vendryes, grand lecteur des moralistes classiques, celui-ci n'entretient pas de rapport direct avec le sujet de l'article Sur la dénomination dans le dossier duquel il est placé. Une hypothèse probable est que cet aphorisme réfère à l'article de presse sur l'enseignement des dialectes locaux. Or, comme cet article ne semble pas non plus avoir de rapport avec le travail en cours, sa présence s'expliquerait par la proximité chronologique avec le premier article. Celui-ci, en revanche, aborde la question qui est cœur de l'article Sur la dénomination : la relation entre le vocabulaire et les entités extralinguistiques. Les coupures de presse, assez nombreuses dans le fonds Vendryes, ne sont donc pas là par hasard mais semblent bien servir à alimenter la réflexion. Dans le dossier Sur la dénomination, la position liminaire de l'article de presse pourrait indiquer qu'il a joué le rôle d'amorce de l'écriture : l'article du Monde soulevant le problème de la dénomination en latin d'entités extralinguistiques modernes aurait suscité la réflexion sur l'arbitraire du signe développée dans l'article. Si tel est le cas, cela expliquerait la prise de position tardive de Vendryes dans la controverse sur l'arbitraire du signe: plus de dix ans après les objections formulées par Pichon et Benveniste et les réponses de Bally, Frei et Sechehaye ${ }^{20}$.

Vendryes a évidemment à l'esprit cette controverse lors de l'écriture de l'article. L'ensemble des manuscrits du dossier est consacré à la reformulation des arguments des adversaires de l'arbitraire du signe et à leur réfutation. Après les deux coupures de presse, figure d'ailleurs un feuillet où le linguiste dresse la liste de la bibliographie autour de cette question (fig. 5). Un autre feuillet, un peu plus loin dans le dossier, est consacré à cet inventaire bibliographique (fig. 6). 


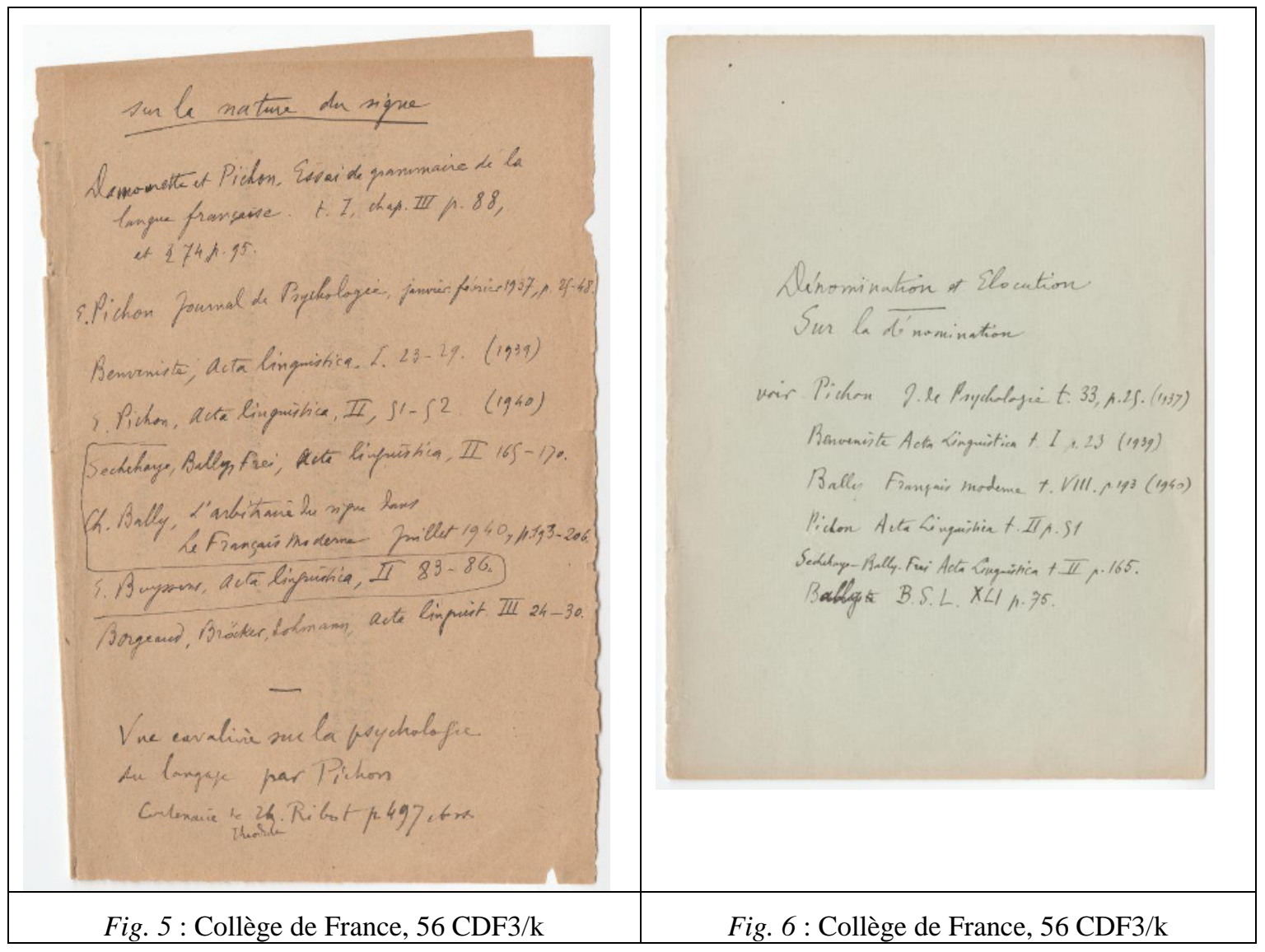

La liste bibliographique sera insérée dans le texte publié :

$*^{\star} *$

Sur ce caractère arbitraire du signe, une controverse s'est élevée naguère, qui serait grave, si elle ne reposait en fin de compte sur un fâcheux malentendu. On en trouvera le détail dans une série d'articles, publiés successivement entre 1937 et 1940 par le Journal de Psychologie, t. XXXIII, p. 25-48, les Acta linguistica, t. I, p. 23-29, t. II, p. 51-52, 83-86, 165-170, t. III, p. 24-30, le Français moderne, juillet 1940 , p. 193-206, le Bulletin de la Société de Linguistique, t. XLI, p. 75 , etc. Il est indispensable de bien préciser la nature du débat.

\section{(Vendryes $1952: 6$ )}

La confrontation de ces trois listes appelle plusieurs observations. La première concerne les variations dans la manière dont le linguiste désigne, chemin faisant, son travail : «Sur la nature du signe », «Dénomination et Élocution», «Sur la dénomination». Ces variations révèlent un possible travail théorique sur la notion d'élocution, mise en miroir avec la dénomination, mais qui n'est conservé ni dans le dossier manuscrit, ni dans l'article définitif. Le contenu des listes bibliographiques lui-même s'avère également problématique car aucune des deux listes manuscrites ne correspond en tout point à celle publiée. La seconde (fig. 6) ne compte que six des huit titres conservés dans la version publiée. La première (fig. 5) est plus complète puisqu'elle inventorie neuf titres; elle peut être considérée comme postérieure car elle témoigne d'un souci de classement chronologique avec le déplacement de l'article de 
Buyssens entre celui Pichon et celui de Sechehaye, Bally et Frei paru dans le même volume des Acta Linguistica. Ce souci chronologique qui n'apparaît pas dans l'autre liste manuscrite sera maintenu dans la liste publiée. Pour autant, un titre manque par rapport à la liste définitive et surtout deux titres ne sont pas repris dans l'article définitif, à savoir l'Essai de grammaire de la langue française de Damourette et Pichon et la «Vue cavalière de la psychologie du langage » publiée par Pichon pour le centenaire de Théodule Ribot, alors que même la discussion de ces deux derniers textes occupe une place très importante dans les manuscrits. Ce sont curieusement les textes les plus discutés dans les manuscrits qui sont omis dans la liste bibliographique de l'article publié.

Un dernier détail, dans la comparaison de ces listes, a son importance : c'est l'effacement des noms propres dans la présentation finale de la bibliographie. Ce détail va de pair avec le traitement anonyme, dans Sur la dénomination, des argumentations de Benveniste et de Pichon. Le nom de Pichon, en particulier, omniprésent dans les manuscrits préparatoires, n'apparaît pas une seule fois dans l'article publié. Les seuls noms propres qui sont mentionnés sont ceux de Saussure et de Meillet parce qu'ils font partie des «éminents linguistes » qui ont «accepté » et «proclamé à maintes reprises » le principe de l'arbitraire (Vendryes, 1952 : 6). Les contradicteurs, quant à eux, ne sont jamais désignés nommément. Il est question seulement d' «autres linguistes, non moins éminents qui se sont refusés à admettre » l'arbitraire (pour désigner Benveniste) et « des psychologues » (pour Pichon).

L'examen des notes rédigées dans le dossier révèle que la défense de l'arbitraire du signe s'élabore surtout, dans les manuscrits, par réfutation. Les six ensembles textuels rédigés ont en commun d'intégrer dans leur argumentation soit une citation, le plus souvent de Pichon, soit la reformulation d'un argument adverse. Prenons l'exemple de cette note (fig. 7) qui s'ouvre sur une citation de Pichon recopiée textuellement bien que sans guillemet, avec mention de la référence :




Au feuillet suivant (recto : fig. 8 et verso : fig. 9), Vendryes adopte une disposition dialogale. Il oppose topographiquement les positions en présence : il isole avec une accolade la thèse du CLG - «Le signifiant est arbitraire par rapport au signifié avec lequel il n'a aucune attache dans la réalité » (Saussure, 1916 : 101) - puis, après la mention «Pichon réplique », il recopie un extrait du paragraphe 74, intitulé « Le signe n'est pas arbitraire », du premier volume des Mots à pensée de Damourette et Pichon (1930 : 97) :

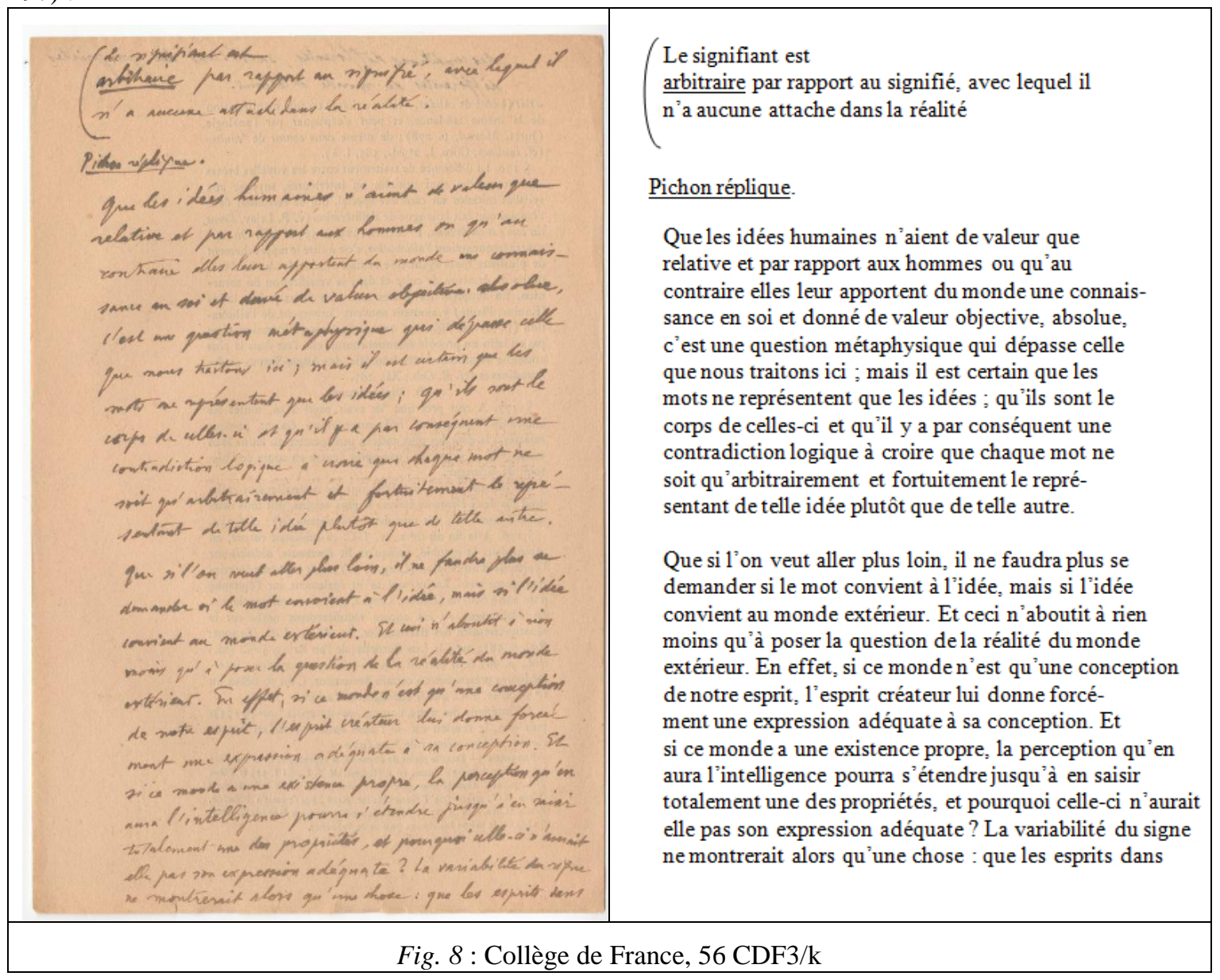

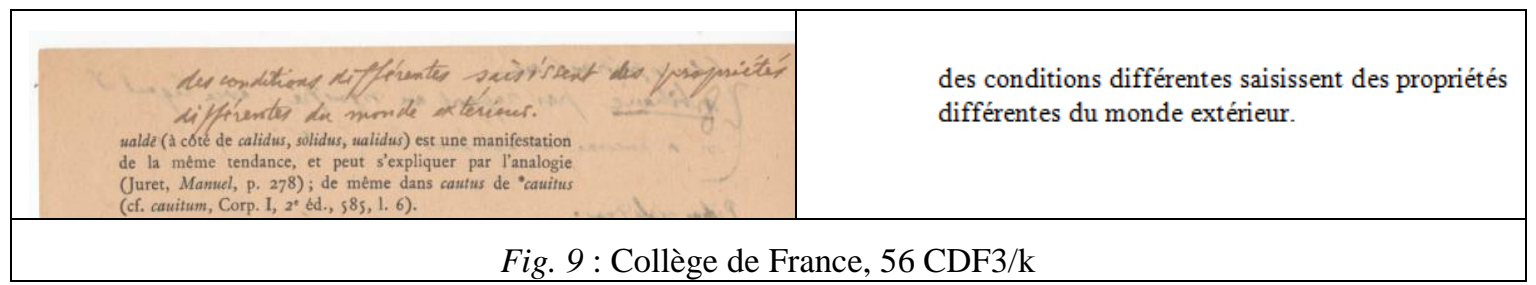

Sur le feuillet suivant (fig. 10), il garde la même disposition mais il inverse les pôles argumentatifs : le haut du feuillet est réservé aux arguments contre l'arbitraire, les bas aux arguments en sa faveur. 


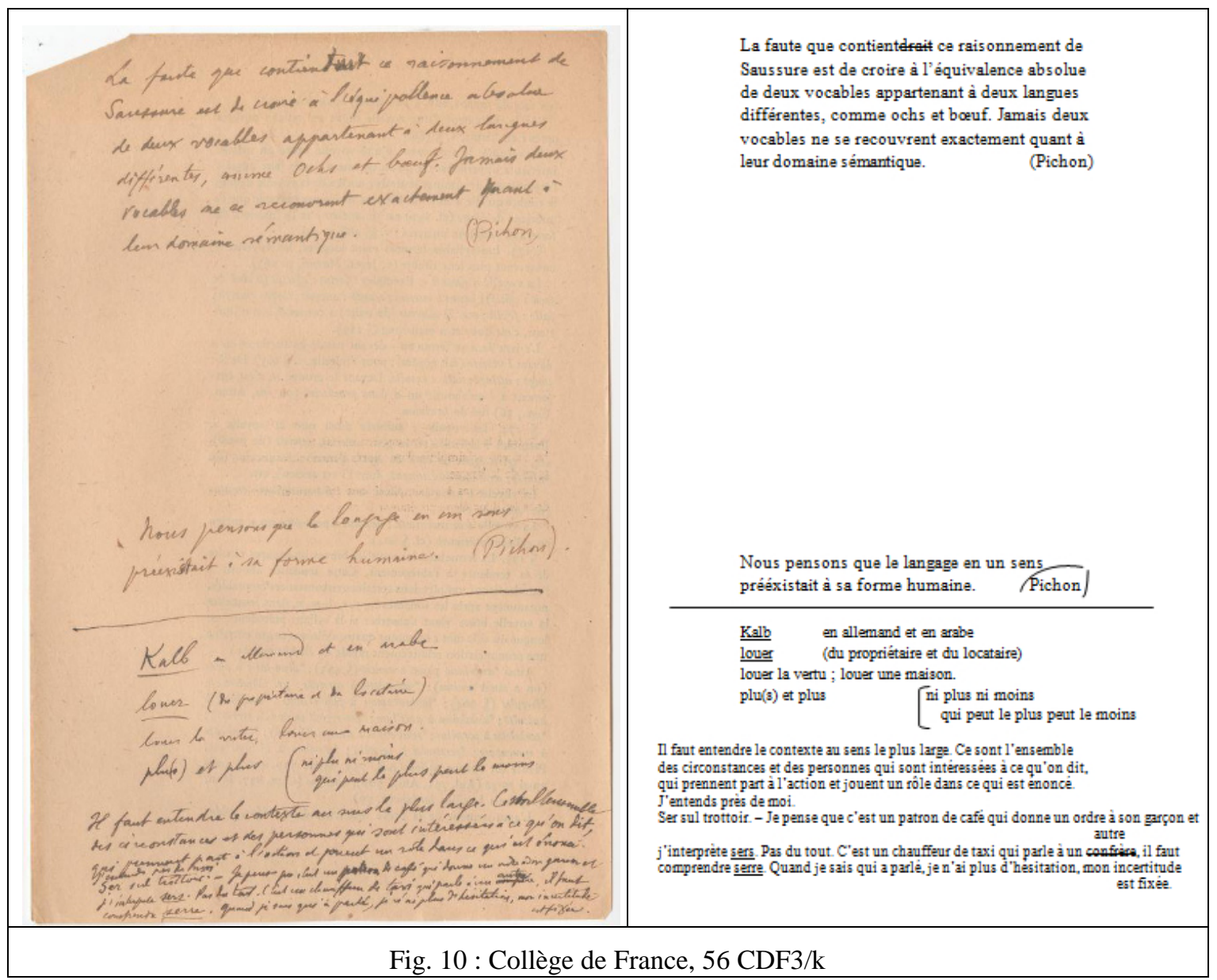

La disposition topographique de l'écrit sur ce feuillet participe de la recherche d'arguments : en haut sont recopiés les critique des Damourette et Pichon (1930 : 96-97) contre l'exemple maladroit ochs et bouf du $C L G$, le bas est consacré à la recherche d'arguments opposés. Le passage inspire à Vendryes les arguments inverses de ceux de Saussure, c'est-à-dire des exemples d'homophonie translinguistique (kalb en allemand et en arabe) et d'homophonies intralinguistiques («louer la vertu ; louer une maison »). Certains de ces exemples seront intégrés dans l'article définitif au service de l'arbitraire, même s’ils sont évidemment en bute aux mêmes objections que la démonstration du CLG sur ochs et bouf.

Ce qui nous intéresse ici est le processus par lequel s'élaborent les arguments qui seront développés dans l'article. L'écriture dialogale à laquelle recourt Vendryes exploite les ressources de la page, le trait horizontal du second feuillet équivalant au «Pichon réplique » du premier feuillet, ou plus exactement à un «je réplique ». C'est au terme d'une construction en chiasme - pour l'arbitraire (CLG) / contre l'arbitraire (Pichon); contre l'arbitraire (Pichon) / pour l'arbitraire (Vendryes) - que le linguiste passe de l'état des lieux de la question à la formulation de ses propres arguments.

Ces documents préparatoires révèlent le dialogue souterrain avec les textes de Pichon et de Benveniste qui préside à l'écriture de Sur la dénomination et qui s'avère, au final, feutré et allusif dans l'article publié. Les quelques feuillets examinés ne relèvent pas du simple travail de collecte de données préalables à une rédaction. Même dans le recopiage des citations qui seront discutées, deux opérations se font jour, qui témoignent de la réflexion déjà à l'œuvre : la sélection et l'agencement. La sélection car Vendryes ne recopie pas l'intégralité des citations de Pichon; il choisit les passages de l'argumentation, en omettant ici ou là une phrase, spécifiquement celles où apparaît la terminologie spécifique de Damourette et Pichon ${ }^{21}$. L'agencement car la collecte est aussi une confrontation de données, article de 
presse ou citation, d'où naît la pensée. C'est avec ces deux opérations que s'amorce l'écriture. Si l'on ne saurait tirer de l'étude d'un seul dossier des enseignements prématurés, il semble qu'une des spécificités de la méthode de Vendryes réside dans le point de départ de l'écriture pris dans les écrits d'autrui, que ce soit au niveau global de l'article avec la coupure de presse, ou au niveau local de chaque note de travail.

\section{Conclusions}

Au moment de conclure, les apports de cette plongée dans les manuscrits de travail de Joseph Vendryes peuvent sembler bien minces. Plus que des révélations - l'existence d'un projet avorté sur l'esthétique du langage, la préparation de l'article de 1952 -, c'est un gain heuristique que l'on peut retirer de ce type de documents et, concernant Vendryes, un déplacement du regard. Avec les manuscrits de travail, ce sont certaines des évidences répétées sur cette «figure de transition » qui se trouvent déplacées : un rapport au Cercle Linguistique de Prague plus complexe et plus approfondi qu'on ne l'a souvent dit, un intérêt pour une problématique - le rapport entre langage et esthétique - rare dans le contexte français de l'époque, une façon spécifique de poser un problème de linguistique générale.

La prise en compte de ces documents de travail s'inscrit dans le courant de recherche actuel qui s'intéresse aux actes quotidiens des chercheurs, et spécifiquement à leurs gestes d'écriture. Qu'elles émanent de linguistes (Mondada, 2005, Doquet-Lacoste, 2009), de sociologues (Lefebvre, 2013, Bert, 2014) ou d'historiens des sciences (Jacob, 2011, Waquet, 2015), toutes ces approches ont en commun de considérer les écrits quotidiens des chercheurs comme des observatoires privilégiés de la science en train de se faire. Le manuscrit de travail d'un savant se trouve, pour l'historien de la linguistique, au carrefour de différentes approches : conceptuelle et sociale. Il peut être investi par des questionnements de recherche qui relèvent aussi bien d'une histoire interne que d'une histoire externe. Grâce aux manuscrits de savants, la description et la théorisation des langues et du langage peut être appréhendée comme une pratique discursive ancrée dans des processus scripturaux. Il en va ainsi de la linguistique générale au début du XXe siècle. Qu'est-ce finalement que généraliser? De nombreux travaux (Koerner, 1988, Auroux, 1988 et 2000, Normand, 2000, Colombat, Fournier \& Puech, 2010 : 209-215) ont souligné le flou qui entoure la notion de la linguistique générale, mais peu se sont consacrés aux méthodes concrètes correspondant à ce champ. Or, les différentes conceptions de la linguistique générale que développent un Saussure, un Meillet, un Vendryes, un Sapir ou un Hjelmslev ne sont sans doute pas étrangères aux différentes façons dont chacun procède pour dégager un savoir à caractère général sur les langues. Cellesci restent encore à explorer car si « la linguistique générale » est au début du XXe siècle un syntagme polysémique qui recouvre des démarches diverses, celles-ci se font toutes un stylo à la main.

\section{Références bibliographiques}

Auroux, S. (1988). La notion de linguistique générale. Histoire Epistémologie Langage, 10/2, 37-56.

Auroux, S. (2000). Les antinomies méthodologiques. In : Auroux, S. (dir.), Histoire des idées linguistiques. Tome 3. L'hégémonie du comparatisme. Liège et Bruxelles : Mardaga, 409-440.

Bally, C. (1932). Linguistique générale et linguistique française. Paris : Ernest Leroux.

Barberousse, A. et Pinon, L. (dir.) (2003). Genesis, 20 : L'écriture scientifique.

Bataillon, M. (1960). Éloge funèbre de M. Joseph Vendryes, membre de l'Académie. Comptes rendus des séances de l'Académie des Inscriptions et Belles-Lettres, 104e année $n^{\circ} 1,46-53$.

Benveniste, É. (1960). Joseph Vendryes (1875-1960). Bulletin de la Société de linguistique de Paris, 55, 1-9.

Benveniste, É. (2011). Baudelaire, éd. C. Laplantine. Limoges : Lambert-Lucas.

Bert, J.-F. (2014). Qu'est-ce qu'une archive de chercheur? Marseille: OpenEdition Press : http://books.openedition.org/oep/438.

Bouquet, S. (1988). Les archives d'Antoine Meillet au Collège de France. Présentation et catalogue provisoire. Archives et documents de la SHESL, 8, 113-143. 
Brunet, E. (2011). Les manuscrits d'Émile Benveniste. Histoire et échantillon de matériaux inédits à explorer. Cahiers Ferdinand de Saussure, 64, 211-228.

Chabanne, J.-C. (1993). Queneau et la linguistique (2). Queneau lecteur de J. Vendryes, de la linguistique à la philosophie du langage. Temps mêlés, documents Queneau, 150-157-160, 39-55.

Charle, C. (1986). Les professeurs de la faculté des lettres de Paris. Dictionnaire biographique 1909-1939. Paris : Éditions du CNRS.

Chepiga, V. (2012). La préparation d'un ouvrage inachevé : «La glottologie » de Lucien Tesnière. Genesis, 35, 8799.

Chepiga, V. et Sofia, E. (dir.) (2014). Archives et manuscrits de linguistes. Louvain-la-Neuve: AcademiaL'Harmattan.

Chevalier, J.-C. et Encrevé, P. (2006). Combats pour la linguistique, de Martinet à Kristeva. Essai de dramaturgie épistémologique. Paris : ENS éditions.

Chevalier, J.-C. (2013). Linguistique et philologie française devant l'analyse des structures (1876-1956). Les dossiers de HEL, $3:$ http://htl.linguist.univ-paris-diderot.fr/num3/cheval.pdf

Chudak, H. (2011). La pensée métapoétique de Blaise Cendrars et ses rapports avec les théories de Joseph Vendryes. In : Russo, M. T. (dir.) Blaise Cendrars et ses contemporains. Entre texte(s) et contexte(s). Palerme : Flavicco, 83-92.

Colombat, B., Fournier, J.-M. et Puech, C. (2010). Histoire des idées sur le langage et sur les langues. Paris : Klincksieck.

Corcy-Debray, S. (2001). Jérôme Carcopino, un historien à Vichy. Paris : L’Harmattan.

Damourette, J. et Pichon, É. (1930). Des mots à la pensée. Essai de grammaire de la langue française. Tome 1. Paris : D'Artrey.

Desmet, P. et Swiggers, P. (1996). Vendryes, Joseph (Jean-Baptiste-Marie). In : Stammerjohann, H. (dir.). Lexicon grammaticorum. Tübingen : Max Niemeyer, 962-964.

D’Iorio, P. et Ponton, O. (dir.) (2003). Genesis, 22 : Philosophie.

D’Ottavi, G. (2012). Genèse d'un écrit saussurien : de la «théosophie » à une approche de la subjectivité. Genesis, $35,129-141$.

Doquet-Lacoste, C. (dir.) (2009). Langage et société, 127/1 : Écritures scientifiques. Carnets, notes, ébauches.

Engler R. (1962). Théorie et critique d'un principe saussurien : 1'arbitraire du signe. Cahiers Ferdinand de Saussure, 19, 5-66.

Fenoglio, I. (2009). Les notes de travail d'Émile Benveniste: où la pensée théorique naît via son énonciation. Langage et société, 127, 23-49

Fenoglio, I. (dir.) (2012). Genesis, 35 : Le geste linguistique.

Fenoglio, I. (2012a). Genèse du geste linguistique : une complexité heuristique. Genesis, 35, 13-39.

Forel, C. (2008). La linguistique sociologique de Charles Bally. Genève : Droz.

Godel, R. (1957). Les sources manuscrites du Cours de linguistique générale. Genève : Droz.

Granucci, F. (1992). Complementi a « Pour un manuel de linguistique générale » di Antoine Meillet, éd. F. Granucci. Studi e saggi linguistici, 32, 13-232.

Greimas, A.-J. (1956). L'actualité du saussurisme. Le français moderne, 24, 191-203.

Gueslin, A. (dir.) (1993). Les facs sous Vichy. Étudiants, universitaires et Universités de France pendant la seconde guerre mondiale. Clermont Ferrand : Publication de l'Institut d'Etude du Massif Central.

Haudricourt, A.-G. (2010). Des gestes aux techniques. Essai sur les techniques dans les sociétés pré-machinistes, éd. J-F Bert. Paris - Versailles : Éditions de la Maison des sciences de l'homme - Éditions Quae.

Havránková, M. et Petkevič, V. (2014), Pražská škola v korespondenci. Dopisy z let 1924-1989. Prague : Karolinum. 
Jacob, C. (dir.) (2011). Lieux de savoir 2. Les mains de l'intellect. Paris : Albin Michel.

Koerner, K. (1988). Meillet, Saussure et la linguistique générale. Histoire Epistémologie Langage, 10/2, 57-73.

Lefebvre, M. (dir.) (2013). Sciences de la Société, 89: L'infra-ordinaire de la recherche. Archives, mémoires et patrimoine scientifique: http://sds.revues.org/193

Loicq, J. (1962-1963). Bibliographie de J. Vendryes. Complément pour 1952-1960. Études celtiques, 10, 349-353.

Mathieu, G. (2011). La Sorbonne en guerre (1940-1944) suivi de Journal de la Libération de Versailles, éd. J.-M. Mathieu. Paris : L'Harmattan.

Martin, J.-P. (1998). La bande sonore. Essai sur le roman de la voix. Paris : José Corti.

Meillet, A. (1922). Jespersen (Language), Sapir (Language), Vendryes (Le langage) [compte rendu]. Bulletin de la Société de linguistique de Paris, 23/2, 2-9.

Meillet, A. (1995). Pour un manuel de linguistique générale. Edizione di manoscritti inediti conservati al Collège de France, éd. F. Granucci. Rome : Atti della Academia Nazionale dei Lincei.

Meillet, A et Vendryes, J. (1924). Traité de grammaire comparée des langues classiques. Paris : Honoré Champion.

Mirambel, A. (1960). Joseph Vendryes (1875-1960). Vie et Langage, 99, 295-299.

Mondada, L. (2005). Chercheurs en interaction : comment émergent les savoirs. Lausanne : Presses polytechniques et universitaires romandes.

Mukarovsky, J. (1929). Rapports de la ligne phonique avec l'ordre des mots dans les vers tchèques. Travaux du Cercle Linguistique de Prague, 1, 121-139.

Mukarovsky, J. (1931). La phonologie et la poétique. Travaux du Cercle Linguistique de Prague, 4, 278-288.

Nerlich B. (2001). The development of semasiology in Europe : A survey from the second half of the 19th to the first third of the 20th century. In : Auroux, S., Koerner, K., Niederehe, H.-J. (dir.) History of the language sciences, vol. 2. Berlin - New York : Walter de Gruyter, 1600-1611.

Normand, C. (2000). La généralité des principes. In: Auroux, S. (dir.), Histoire des idées linguistiques. Tome 3. L'hégémonie du comparatisme. Liège et Bruxelles : Mardaga, 463-471.

Puech, C. (2000). Notice Langage. Introduction linguistique à l'histoire. In : Colombat, B. (dir.), Corpus des textes linguistiques fondamentaux. Tome 2. HEL Hors-série 3, 458-460.

Saussure, F. de ([1916] 1967). Cours de linguistique générale, publié par C. Bally et A. Sechehaye, éd. T. De Mauro. Paris : Payot.

Saussure, F. de (1957). Cours de linguistique générale (1908-1909). Introduction (d'après des notes d'étudiants), éd. R. Godel. Cahiers Ferdinand de Saussure, 15, 3-103.

Saussure, F. de (1995). Phonétique. Il manoscritto di Harvard Houghton Library bMS Fr 266 (8), éd. M. P. Marchese. Florence : Unipress.

Saussure, F. de (1968-1974). Cours de linguistique générale. Édition critique par R. Engler. Wiesbaden : Otto Harrassowitz.

Saussure, F. de (2002). Écrits de linguistique générale, éd. S. Bouquet et R. Engler. Paris : Gallimard.

Saussure, F. de (2005). Notes préparatoires pour le cours de linguistique générale ; 1910-1911, éd. D. Gambarara et C. Mejía Quijano, Cahiers Ferdinand de Saussure, 58, 83-290.

Saussure, F. de (2011). Science du langage, éd. R. Amacker. Genève : Droz.

Saussure, F. de (2013). Anagrammes homériques, éd. P.-Y. Testenoire. Limoges : Lambert-Lucas.

Singer, C. (1992). Vichy, l'Université et les juifs. Paris : Les Belles Lettres.

Sofia, E. (2012). Comment écrire pour transmettre ? Modalités argumentatives chez Saussure. Genesis, 35, 147-160.

Sofia, E. (2015). La « collation Sechehaye» du Cours de linguistique générale de Ferdinand de Saussure : édition, introduction et notes. Leuven : Peeters. 
Starobinski, J. (1971). Les mots sous les mots. Les anagrammes de Ferdinand de Saussure. Paris : Gallimard.

Swiggers, P. (1991). Les archives d'Antoine Meillet au Collège de France : additions et corrections à l'inventaire. Bulletin de la Société de linguistique de Paris, 86-1, 367-370.

Swiggers, P. (1991a). Notes sur la linguistique générale en 1921-1922. Avec l'édition de deux lettres de Joseph Vendryes à Edward Sapir. Beiträge zur Geschichte der Sprachwissenschaft, 1, 185-191.

Testenoire, P.-Y. (2010). Genèse d'un principe saussurien: la linéarité. Recto-Verso, 6 : http://www.revuerectoverso.com/spip.php?article179

Testenoire, P.-Y. (2013). Ferdinand de Saussure à la recherche des anagrammes. Limoges : Lambert-Lucas.

Testenoire, P.-Y. (2014). Les manuscrits de Joseph Vendryes : premier état des lieux. In : Chepiga, V. et Sofia, E. (dir.). Archives et manuscrits de linguistes. Louvain-la-Neuve : Academia-L'Harmattan, 77-98.

Testenoire, P.-Y. (2015). Présentation et inventaire des archives de Joseph Vendryes au Collège de France, Études celtiques, $41,245-270$.

Troubetzkoy, N. S. (2006). Correspondance avec Roman Jakobson et autres écrits, éd. P. Sériot, trad. du russe par P. Sériot et M. Schönenberger. Lausanne : Payot.

Valin, R. (1971). Avertissement. In: Guillaume, G. Leçons de linguistique de Gustave Guillaume, 1948-1949. Québec - Paris : Presses de l'Université Laval - Klincksieck, 49-67.

Vendryes, J. ([1921] 1968). Le langage. Introduction linguistique à l'histoire. Paris : Albin Michel.

Vendryes, J. (1921). Le caractère social du langage et la doctrine de Ferdinand de Saussure. Journal de psychologie, $18,617-624$.

Vendryes, J. (1930). La poésie galloise des XIIe-XIIIe siècles dans ses rapports avec la langue. Oxford: The Clarendon Press.

Vendryes, J. (1951). Choix d'études linguistiques et celtiques. Paris : Klincksieck.

Vendryes, J. (1952). Sur la dénomination. Bulletin de la Société de linguistique de Paris, 48, 1-13.

Waquet, F. (2015). L'ordre matériel du savoir Comment les savants travaillent : XVI ${ }^{e}-X X I^{e}$ siècles. Paris : Éditions du CNRS.

${ }^{1}$ Indice révélateur de ce mouvement de recherche: l'existence, au sein du laboratoire ITEM, d'une équipe «Génétique du texte et théories linguistiques » dirigée par Irène Fenoglio qui consacre l'ensemble de ses activités à l'étude des manuscrits de travail de linguistes. Cette équipe a pour objectif «la compréhension de la genèse des concepts et des théories linguistiques, par l'observation des manuscrits d'auteurs dans le domaine des sciences du langage » (site de l'ITEM : http://www.item.ens.fr/index.php?id=579254, consulté le 21/02/2016).

${ }^{2}$ C'est le meilleur témoin choisi par Godel dans son édition de 1957 de l'introduction du deuxième cours (Saussure, 1957). Rudolf Engler, quant à lui, ne collationne pas les versions en un seul texte et opte pour une confrontation synoptique des différents cahiers d'étudiants. Son coup de génie est de renverser la présentation canonique des éditions philologiques : à la verticalité hiérarchisante du texte et de son apparat critique en notes, il substitue une horizontalité heuristique.

${ }^{3}$ Parmi les éditions des vingt dernières années, citons celles de Marchese (Saussure, 1995), d'Engler et de Bouquet (Saussure, 2002), de Gambarara et de Mejia Quijano (Saussure, 2005), d'Amacker (Saussure, 2011), de Testenoire (Saussure, 2013) et de Sofia (2015).

4 L'inventaire est également accessible dans le catalogue en ligne des archives du Collège de France: https://salamandre.college-de-france.fr. On trouvera également dans Testenoire (2014) un premier aperçu des spécificités des archives de Joseph Vendryes ainsi qu'un historique du fonds plus détaillé qu'il ne l'est ici. Nous remercions le service des archives du Collège du France de nous avoir autorisé à citer et à reproduire des manuscrits du fonds Joseph Vendryes.

${ }^{5}$ Nous tenons à remercier M. Georges Vendryes, fils du linguiste, qui nous a aimablement communiqué des copies des manuscrits en sa possession et qui nous a autorisé à les citer. 
${ }^{6}$ Cette tripartition est proposée par Vendryes lui-même. Invité en 1952 par la Société de linguistique de Paris à rééditer une sélection de ses articles, il choisit de les réunir dans trois sections qu'il intitule Linguistique générale, Grammaire comparée et Antiquités celtiques. Sur la base de la liste de ses publications (Vendryes, 1952: 335-350 et Loicq, 1962-1963), il est possible de donner une idée indicative des proportions entre ces trois champs : plus de la moitié des publications de Vendryes portent sur les langues celtiques, environ un tiers sur d'autres langues indoeuropéennes, moins de $20 \%$ concerne la linguistique générale.

${ }^{7}$ Cf. respectivement, à titre d'exemple : Nerlich (2001: 1606), Chevalier (2013:3), Koerner (1988:62) et Martinet (in Chevalier \& Encrevé, 2006 : 61).

${ }^{8}$ Ce passage concorde avec le témoignage de Benveniste : «Pour celui qui a fait son apprentissage avec ces deux maîtres [Meillet et Vendryes], c'est une douceur de les évoquer ensemble, tels qu'ils nous apparaissaient à nous débutants. Si bien ils s'entendaient, malgré leurs dissemblances, que la linguistique prenait à nos yeux, selon ses parties, l'image de l'un, tantôt de l'autre et qu'elle nous semblait se configurer entièrement dans leurs traits complémentaires. À travers Vendryes, c'était encore pour l'essentiel l'enseignement de Meillet qu'on recevait. » (Benveniste, $1960: 3$ ).

${ }^{9}$ Derrière l'identité des titres Le Langage/Language, les sous-titres sont révélateurs de la diversité des orientations : Introduction to the Study of Speech pour Sapir, Introduction linguistique à l'histoire pour Vendryes, Its Nature, Development and Origin pour Jespersen, cf. Swiggers 1991a.

${ }^{10}$ Nous remercions Catherine Pinon pour les informations concernant la traduction arabe du Langage.

${ }^{11}$ V. Chabanne, 1993, Martin, 1998 : 95-99 et Chudak, 2011.

${ }^{12}$ Sur le rôle de Joseph Vendryes en tant que doyen durant l'occupation, on consultera Singer (1992: 175 et 202203), Gueslin (1993), Corcy-Debray (2001 : 232) et Matthieu (2011). Les historiens s'accordent à lui reconnaître « une attitude résistante » à ce poste (Charle, 1986 : 210). Elle lui vaudra une incarcération à la prison de Fresnes en avril 1942, puis d'être suspendu de ses fonctions le $1^{\text {er }}$ avril 1944. Vendryes sera réintégré à son poste à la libération, le 20 août 1944.

${ }^{13}$ Marcel Bataillon auquel il reviendra de prononcer l'éloge funèbre de Vendryes à l'Académie des Inscriptions et Belles-Lettres fait allusion à ce texte et en utilise de nombreux extraits (Bataillon 1960).

${ }^{14}$ La Collection philosophique d'Alcan est la Bibliothèque de Philosophie contemporaine, ancêtre de l'actuelle collection Quadrige des Presses Universitaires de France. Elle accueille dans ces années de l'entre-deux guerres, les ouvrages majeurs de l'idéalisme et du spiritualisme français - Brunschvicg, Le Progrès de la conscience dans la philosophie occidentale (1927), Bergson, Durée et simultanéité (1922), Les deux sources de la morale et de la religion (1932) -, mais aussi de l'école française de sociologie et d'anthropologie : Lévy-Bruhl, La mentalité primitive (1922), les éditions posthumes de Durkheim, Éducation et sociologie (1922), Sociologie et philosophie (1924), Goblot, La barrière et le niveau : étude sociologique sur la bourgeoisie française moderne (1925)...

${ }^{15}$ Henri Delacroix (1873-1937), professeur de psychologie à la Sorbonne, est l'auteur de plusieurs ouvrages dans cette collection : La religion et la foi (1922), Le langage et la pensée (1924), Psychologie de l'art (1927)... Doyen de la faculté des lettres depuis le $1^{\mathrm{e}}$ septembre 1928, Delacroix a pour accesseur Vendryes qui lui succèdera en 1937.

${ }^{16}$ Il s'agit de la conférence sur « la poésie galloise des XIIe-XIIIe siècles dans ses rapports avec la langue » donnée à Oxford dans le cadre de The Zaharoff Lecture et dont le texte est publié dans Vendryes (1930).

${ }^{17}$ Affirmation que l'on retrouve dans la nécrologie de Vendryes par André Mirambel : «J. Vendryes admirait aussi les écrivains français plus récents, Guy de Maupassant et sa prose étonnante, Marcel Proust et son «sens aigu de la langue », les poètes symbolistes comme les classiques eux-mêmes; en lettré et en linguiste, il se réjouissait que la phonologie eût, disait-il, «ramené dans les cadres de la linguistique l'étude de l'esthétique du langage ». Ainsi s'édifiait, selon son vœu, un pont entre l'art et la science. » (Mirambel, 1960 : 296-297).

${ }^{18} \mathrm{Cf}$. « le phonème est une unité phonologique non susceptible d'être dissociée en unités phonologiques plus petites et plus simples » (TCLP $4: 311)$.

${ }^{19}$ La correspondance entre Vendryes et les membres du CLP entre 1935 et 1938 qui fait état du projet de la publication de l'article dans les TCLP a récemment été publiée : Havránková \& Petkević 2014 : 710-716. 


\begin{abstract}
${ }^{20}$ L'article d'Engler de 1962 fait le point sur cette controverse autour du principe de l'arbitraire du signe. Il recense entre les années 20 et la fin des années 50 environ soixante-dix prises de positions de linguistes sur cette question.

${ }^{21}$ Voici par exemple le passage des Mots à la pensée recopié par Vendryes fig. 10 : «La faute que nous paraît contenir ce raisonnement de Saussure est de croire à l'équipollence absolue de deux vocables appartenant à deux langues différentes, comme ochs et boeuf. Jamais l'extension de deux sémièmes dans deux idiomes différents n'est exactement la même ; jamais deux vocables ne se recouvrent absolument quant à leur domaine sémantique. » (Damourette et Pichon 1930 : 96). Vendryes omet délibérément la phrase «jamais l'extension de deux sémièmes dans deux idiomes différents n'est exactement la même ».
\end{abstract}

